\title{
Advanced glycation end products induce the apoptosis of and inflammation in mouse podocytes through CXCL9-mediated JAK2/STAT3 pathway activation
}

\author{
JING YU ${ }^{1}$, HAO WU ${ }^{2}$, ZI-YU LIU ${ }^{3}$, QI ZHU ${ }^{1}$, CHANG SHAN $^{1}$ and KE-QIN ZHANG ${ }^{1}$ \\ ${ }^{1}$ Department of Endocrinology of Tongji Hospital, Tongji University School of Medicine, Shanghai 200065; \\ Departments of ${ }^{2}$ Emergency Medicine and ${ }^{3}$ Endocrinology, Changzheng Hospital, \\ Second Military Medical University, Shanghai 200003, P.R. China
}

Received February 29, 2016; Accepted August 9, 2017

DOI: $10.3892 /$ ijmm.2017.3098

\begin{abstract}
Diabetic nephropathy (DN) is a serious and one of the most common microvascular complications of diabetes. There is accumulating evidence to indicate that advanced glycation end products (AGEs), senescent macroprotein derivatives formed at an accelerated rate under conditions of diabetes, play a role in DN. In this study, we found that the serum and urine levels of C-X-C motif chemokine ligand 9 (CXCL9) were significantly elevated in patients with DN compared with healthy controls. Based on an in vitro model of mouse podocyte injury, AGEs decreased the proliferation of podocytes and increased the expression of CXCL9 and C-X-C motif chemokine receptor 3 (CXCR3), and promoted the activation of signal transducer and activator of transcription 3 (STAT3). The knockdown of CXCL9 by the transfection of mouse podoyctes with specific siRNA significantly increased the proliferation and decreased the apoptosis of the podoyctes. Moreover, the levels of inflammatory factors, such as tumor necrosis factor (TNF)- $\alpha$ and interleukin (IL)- 6 were also decreased in the podoyctes transfected with siRNA-CXCL9, accompanied by the increased expression of nephrin and podocin, and decreased levels of Bax/Bcl-2 and activated caspase-3. The knockdown of CXCL9 also led to the inactivation of the Janus kinase 2 (JAK2)/STAT3 pathway. Importantly, the use of the JAK2 inhibitor, AG490, and valsartan (angiotensin II receptor antagonist) attenuated the injury induced to mouse podoyctes by AGEs. On the whole, and to the best of our knowledge, this study demonstrates for the first time that AGEs exert pro-apoptotic and pro-inflammatory effects in mouse podoyctes through
\end{abstract}

Correspondence to: Dr Ke-Qin Zhang, Department of Endocrinology of Tongji Hospital, Tongji University School of Medicine, 1239 Siping Road, Shanghai 200065, P.R. China

E-mail: keqinzhangz@sina.com

Key words: diabetic nephropathy, advanced glycation end products, podocyte, C-X-C motif chemokine ligand 9, Janus kinase-2/signal transducer and activator of transcription 3 the CXCL9-mediated activation of the JAK2/STAT3 pathway. Thus, our data provide a potential therapeutic target for DN.

\section{Introduction}

As one of the most common causes of end-stage renal disease, diabetic nephropathy (DN) accounts for the disability and high mortality rate in patients with diabetes. The incidence of DN in patients with type 1 or 2 diabetes within 20-25 years of the onset of the disease is approximately $25-40 \%$ (1). Although the prevention and treatment of $\mathrm{DN}$ are attracting increasing attention from researchers, the current therapeutic options are far from satisfactory as the intensive therapy of blood glucose and pressure is often difficult to maintain and may increase the risk of hypoglycemia and/or hypotension in patients with diabetes (2). Therefore, the development of novel therapeutic strategies that may specifically target DN is urgently required.

Podocytes are one of the most important components of the glomerular filtration barrier which has specific cytobiological traits and physiological functions. It has been shown that the loss of podocytes is an early characteristic of DN that predicts its progressive course (3), and decreased nephrin expression is observed during the early stages of DN, playing an important role in accelerating the development of DN (4). DN has been reported to be an immunological renal disease; the presence of tumor necrosis factor- $\alpha$ (TNF- $\alpha$ ), interleukin (IL)-1 $1 \beta$, IL-6, interferon- $\gamma$ (INF- $\gamma$ ) and monocyte-chemoattractant protein-1 (MCP-1) has been reported in patients with diabetes and in animal diabetic models (5-7). However, the exact role of podocytes in the inflammatory response has not yet been fully determined.

Various elements, including advanced glycation end products (AGEs), reactive oxygen species (ROS), protein kinase $\mathrm{C}$ (PKC) and the renin-angiotensin system (RAS), are considered to play a role in the development and progression of DN (8). AGE formation in the kidneys may contribute to the progressive alteration in the renal architecture and loss of renal function in patients, resulting in basement membrane thickening and mesangial expansion, hallmarks of DN (9). The activation of AGEs in podocytes leads to multiple pathophysiological effects, including hypertrophy with cell cycle arrest 
and apoptosis, altered migration and in the generation of proinflammatory cytokines (10). Previous studies have reported that AGEs reduce podocyte adhesion via the upregulation of integrin-linked kinase (ILK) expression, which occurs partly through the activation of the RAS in podocytes (11). AGEs are also potent stimulators of chemokine production, including IL-8 [also known as C-X-C motif chemokine ligand (CXCL)8], MCP-1 (CCL2), INF- $\gamma$ inducible protein 10 (IP-10/CXCL10), macrophage inflammatory protein-1 $\alpha$ (MIP-1 $\alpha /$ CCL3) and RANTES (CCL5) (7). The downregulation of CXCL9 and its receptor, CXCR3, suppresses the loss of renal function and may be a potential therapeutic target for human immune-mediated nephritis (12). However, the role of CXCL9 in AGE-induced podocyte injury has not yet been reported to date, at least to the best of our kowledge.

The activation of the Janus kinase (JAK)/signal transducers and activators of transcription (STAT) pathway is an important mechanism through which hyperglycemia contributes to renal damage (13). Similarly, JAK/STAT activation has been reported in rat glomerular cells exposed to high glucose (14) and may be important in glomerular transforming growth factor- $\beta$ (TGF- $\beta$ ) activation in early DN (15). Moreover, angiotensin-convertingenzyme (ACE) inhibitors and angiotensin receptor blockers, including valsartan, which prevent the progression of DN, also prevent JAK/STAT activation in glomerular cells from diabetic rats (16).

In this study, we examined the effect of AGEs on the proliferation and apoptosis of podocytes and on the expression of CXCL9 and the JAK2/STAT3 signaling pathway. We also investigated whether AGE-induced podocytes injury occurs through the CXCL9-mediated activation of the JAK2/STAT3 pathway.

\section{Materials and methods}

Patient samples. Serum and urine samples were obtained from 45 patients with DN admitted to Tongji Hospital, Shanghai, China and subjects in the control group were 45 healthy volunteers. Ethical approval for the study was provided by the independent Ethics Committee of Tongji Hospital. Written informed consent was obtained from all participants in this study. All the experimental procedures were carried out in accordance with the Helsinki Declaration, 1975.

Cell culture and treatment. Mouse podocytes were obtained from the Shanghai Cell Bank, Chinese Academy of Sciences (Shanghai, China) and cultured in RMPI-1640 supplemented with $10 \%$ fetal bovine serum (FBS), 100X penicillin-streptomycin solution and $10 \mathrm{U} / \mathrm{ml} \mathrm{IFN}-\gamma$, and incubated in a humidified atmosphere at $33^{\circ} \mathrm{C}$ with $5 \% \mathrm{CO}_{2}$. Following proliferation to $80 \%$ confluence, the podocytes were cultured in the above-mentioned medium without $10 \mathrm{U} / \mathrm{ml} \mathrm{IFN}-\gamma$ and incubated in a humidified atmosphere at $37^{\circ} \mathrm{C}$ with $5 \% \mathrm{CO}_{2}$ for 10-14 days. To establish the injury model, the podocytes were seeded at $80 \%$ confluence in complete medium (Invitrogen Life Technologies, Carlsbad, CA, USA) containing 10\% FBS. After $24 \mathrm{~h}$, the medium was changed to serum-free medium with AGEs (10,50, 100 and $150 \mathrm{mg} / \mathrm{l})$ at the indicated time points, respectively. In addition to AGE $(150 \mathrm{mg} / \mathrm{l})$ induction, podocytes were also treated with $10 \mu \mathrm{M}$ AG490 or $20 \mu \mathrm{M}$ valsartan (as a positive control) for $48 \mathrm{~h}$.
Cell proliferation assay. Mouse podocytes (1x10 $/$ well) were plated in 96-well plates. Following treatment with AGEs for 12, 24, 48 and 72 h, 10\% cell counting kit-8 (CCK-8, CK04; Dojindo Molecular Technologies, Kumamoto, Japan) diluted in serum-free RMPI-1640 was mixed in each well for a further $1 \mathrm{~h}$. The absorption of each sample was measured at a $450 \mathrm{~nm}$ wavelength using a Labsystems MK3 microplate reader (Thermo Fisher Scientific, Inc., Rockford, IL, USA) to detect cell viability according to the manufacturer's instructions. Cells not treated with AGEs served as the control group.

Transfection with small interfering RNA (siRNA). siRNA targeting CXCL9 (siRNA-CXCL9; Sangon Biotech Co., Ltd., Shanghai, China) was used to knockdown CXCL9 mRNA expression. The cells were transfected with siRNA $(40 \mathrm{nM})$ using Lipofectamine 2000 (Invitrogen, Carlsbad, CA, USA) following the manufacturer's instructions. Non-specific siRNA (Sangon Biotech Co., Ltd.) was used as a negative control (NC), and the selective silencing of CXCL9 was confirmed by real-time PCR. The cells were analyzed at $48 \mathrm{~h}$ following transfection.

Cell apoptosis assay. The analysis of cell apoptosis was performed using flow cytometry and the Annexin V apoptosis detection kit (eBioscience, San Diego, CA, USA). Briefly, the mouse podocytes were plated in 6-well plates at a density of 1x10 $0^{5}$ cells/well and incubated with $195 \mu \mathrm{l}$ Annexin V and $5 \mu \mathrm{l}$ propidium iodide (PI) for $15 \mathrm{~min}$ in the dark at $4^{\circ} \mathrm{C}$. The early apoptotic cells are represented in the lower right quadrant of the FACS histogram, and the late apoptotic cells, which were stained with FITC and PI, emit red-green fluorescence and are represented in the upper right quadrant of the FACS histogram.

Real-time PCR. Total RNA was extracted using TRIzol reagent (Invitrogen Life Technologies) according to the manufacturer's instructions. The complementary DNA was synthesized using a cDNA synthesis kit (Thermo Fisher Scientific, Inc.). The conditions for cDNA synthesis were as follows: $37^{\circ} \mathrm{C}$ for $60 \mathrm{~min}$, followed by $85^{\circ} \mathrm{C}$ for $5 \mathrm{~min}$ and $4^{\circ} \mathrm{C}$ for $5 \mathrm{~min}$. Real-time PCR was performed using SYBR-Green (Takara Biotechnology Co., Ltd., Dalian, China) and data collection was conducted using an ABI 7500 Real-Time PCR system (Applied Biosystems Life Technologies, Foster City, CA, USA). Primers were list as follows: CXCL9 forward, 5'-CACTTCGCTGCTATCTAATTGG-3' and reverse, 5'-TAGGCACTGTGGAAGATTTAGG-3'; CXCR3 forward, 5'-ACCATTACTGTGCCTTAGC-3' and reverse, 5'-TATTTGCCTCTCCCTCTTCTC-3'; STAT3 forward, 5'-GACTCAAAGCCACCTCATTC-3' and reverse, 5'-GCCTT GCCTTCCTAAATACC-3'; and glyceraldehyde 3-phosphate dehydrogenase (GAPDH) forward, 5'-ATCACTGCCA CCCAGAAG-3' and reverse, 5'-TCCACGACGGACACA TTG-3'. GAPDH was used an internal control for normalization. The real-time PCR cycling conditions were as follows: $95^{\circ} \mathrm{C}$ for $10 \mathrm{~min}$, followed by 40 cycles at $95^{\circ} \mathrm{C}$ for $15 \mathrm{sec}$ and $60^{\circ} \mathrm{C}$ for $45 \mathrm{sec}$, and a final extension step of $95^{\circ} \mathrm{C}$ for $15 \mathrm{sec}, 60^{\circ} \mathrm{C}$ for $1 \mathrm{~min}, 95^{\circ} \mathrm{C}$ for $15 \mathrm{sec}$ and $60^{\circ} \mathrm{C}$ for $15 \mathrm{sec}$. Gene expression was calculated using the $2^{-\Delta \Delta \mathrm{Ct}}$ method.

Western blot analysis. Mouse podocytes were seeded at a density of $5 \times 10^{5}$ cells/well in 6 -well plates, cultured 
A

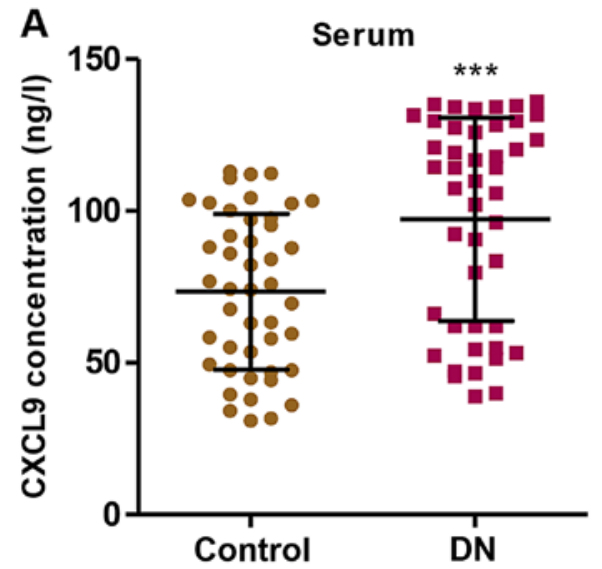

B

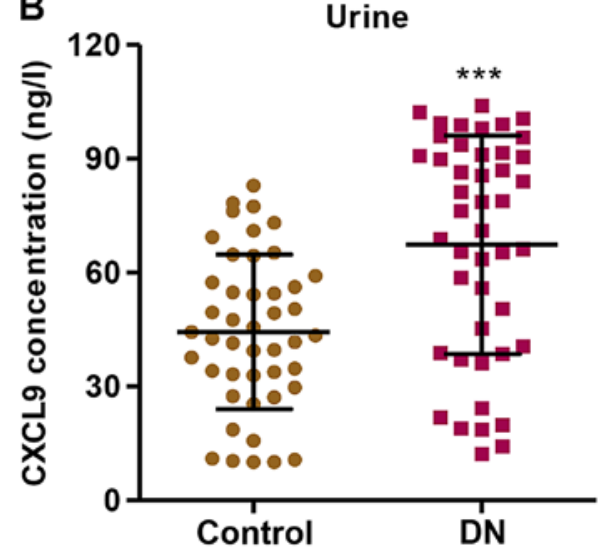

Figure 1. CXCL9 levels in serum and urine of diabetic patients. CXCL9 levels in (A) serum and (B) urine of patients with diabetic nephropathy (DN) were measured by enzyme-linked immunosorbent assay (ELISA). ${ }^{* * *} \mathrm{P}<0.001$ vs. control (healthy subjects).

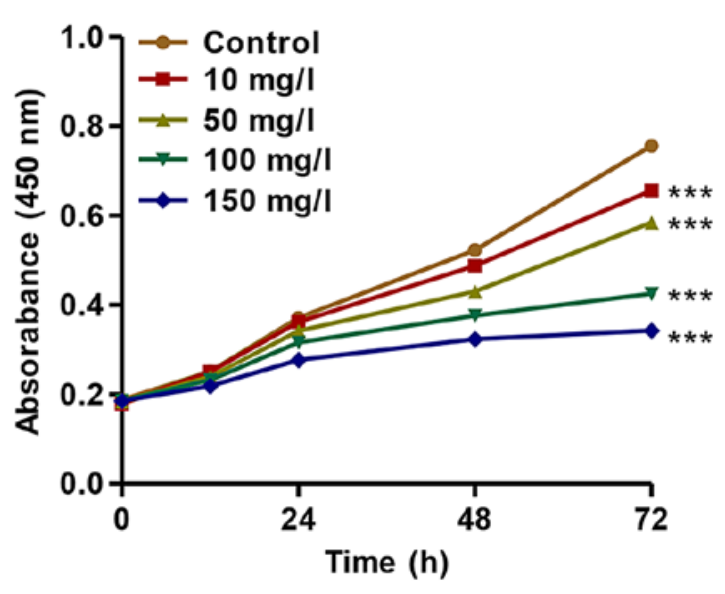

Figure 2. Effect of advanced glycation end products (AGEs) on the proliferation of podocytes. The proliferation of podocytes was measured by cell counting kit-8 (CCK-8) assay at the indicated time points. ${ }^{* * *} \mathrm{P}<0.001$ vs. control.

overnight and then treated with AGEs for 3 or $48 \mathrm{~h}$. Total proteins were isolated from the mouse podocytes and were subjected to $12 \%$ sodium dodecyl sulfate-polyacrylamide gel electrophoresis (SDS-PAGE) and electroblotted onto polyvinylidene fluoride membranes (Roche Diagnostics, Mannheim, Germany). The membranes were first incubated with rabbit monoclonal anti-p-STAT3 (ab76315; 1:1,000; Abcam, Cambridge, MA, USA), anti-JAK2 (\#3230; 1:1,000), anti-pJAK2 (\#3771; 1:1,000) (both from Cell Signaling Technology, Danvers, MA, USA), podocin (ab181143; 1:10,000; Abcam) and anti-GAPDH (\#5174; 1:1,500; Cell Signaling Technology) antibodies, and mouse monoclonal anti-STAT3 (ab119352; $1: 1,000)$ and anti-Bcl-2 (ab117115; 1:400) (both from Abcam) antibodies; as well as rabbit polyclonal anti-CXCL9 (sc-50302; 1:500; Santa Cruz Biotechnology, Inc., Dallas, TX, USA), anti-CXCR3 (ab181013; 1:600; Abcam), anti-Bax (sc-493; 1:300; Santa Cruz Biotechnology, Inc.), anti-caspase-3 (ab44976; 1:500) and anti-nephrin (ab58968; 1:500) antibodies (both from Abcam). The blots were then incubated with goat anti-mouse or anti-rabbit secondary antibodies (A0208 and A0216; 1:1,000; Beyotime Institute of Biotechnology, Haimen, China) and visualized using enhanced chemiluminescence
(ECL; Thermo Fisher Scientific). GAPDH antibody was used as an internal control. The blotting bands were quantified using ImageJ software (National Institutes of Health, Bethesda, MD, USA).

Enzyme-linked immunosorbent assay (ELISA). The TNF- $\alpha$, IL-6 and CXCL9 levels present in the mouse podocytes or in the serum and urine of patients with DN were determined using commercially available murine-specific sandwich ELISA kit following the manufacturer's instructions.

Statistical analysis. Data are expressed as the means \pm SD of triplicate samples. All results were confirmed in at least 3 timeindependent experiments. Statistical analyses were performed using GraphPad Prism 5 software (GraphPad Software, Inc., La Jolla, CA, USA). Statistical analysis was performed using unpaired a two-tailed Student's t-test and one-way ANOVA tests. A value of $\mathrm{P}<0.05$ was considered to indicate a statistically significant difference.

\section{Results}

CXCL9 levels in the serum and urine of patients with DN. In order to examine the role of CXCL9 in DN, we first measured the levels of CXCL9 in both the serum and urine of patients with DN $(n=45)$ and healthy controls $(n=45)$. The CXCL9 concentration was significantly increased in the patients with DN compared with the healthy controls in both serum and urine (Fig. 1). Importantly, a higher concentration of CXCL9 was observed in the serum than in urine. These results suggest that CXCL9 plays an important role in DN; thus CXCL9 was further investigated in subsequent experiments.

Treatment with AGEs inhibits the proliferation of podocytes. To further investigate the association of CXCL9 with DN, we established an in vitro DN model of mouse podocyte injury induced by AGEs. We found that AGEs at various concentrations $(10,50,100$ and $150 \mathrm{mg} / \mathrm{l})$ significantly inhibited the proliferation of mouse podocytes in a concentration- and time-dependent manner (Fig. 2). After $72 \mathrm{~h}$ of incubation, the proliferation of podocytes treated with AGEs (10, 50, 100 and $150 \mathrm{mg} / \mathrm{l})$ was suppressed by $13.31 \pm 0.11 \%, 22.7 \pm 0.17 \%$, 

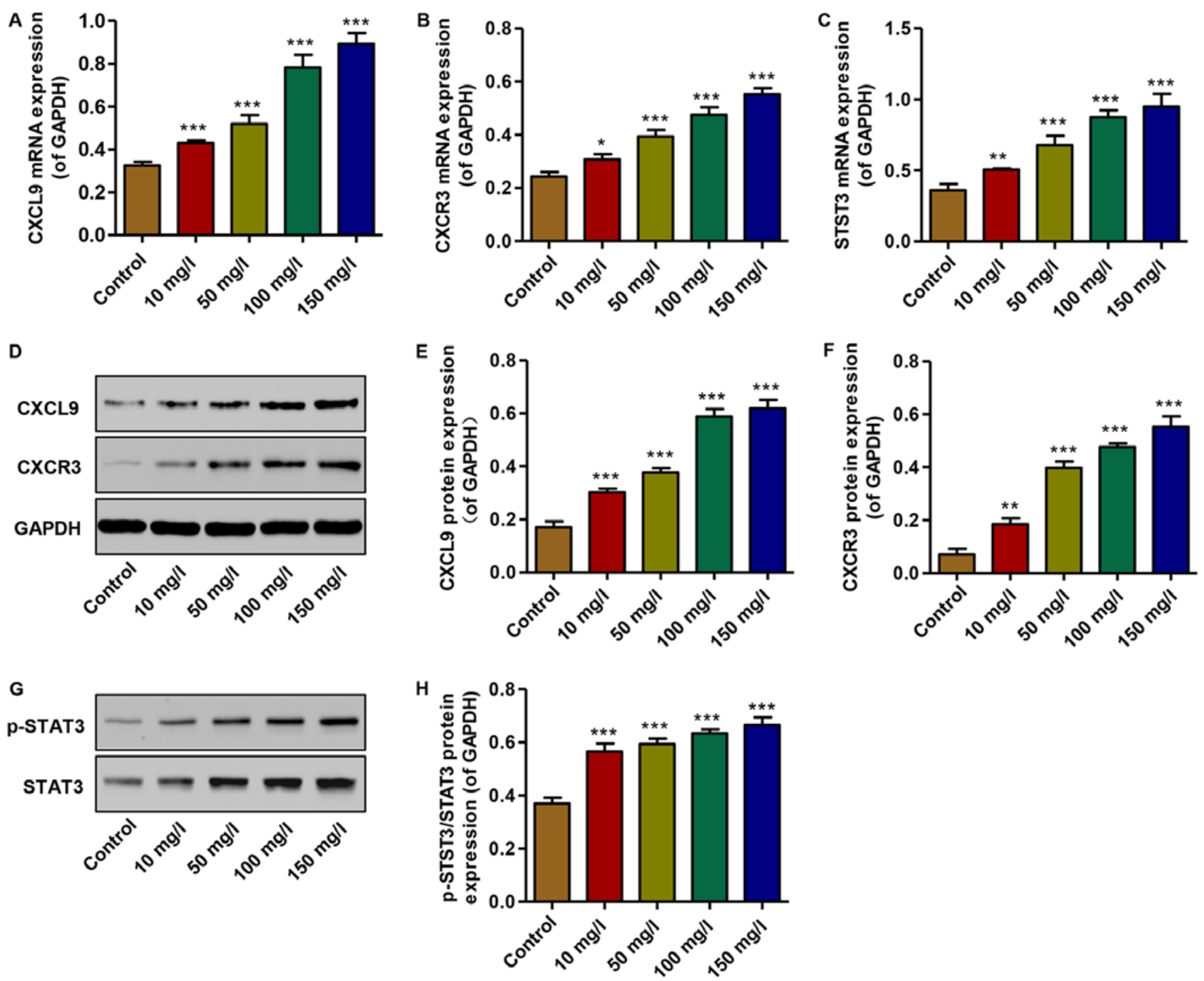

Figure 3. Effect of advanced glycation end products (AGEs) on the expression of CXCL9 and CXCR3 and STAT3 activation. (A-C) The mRNA levels of CXCL9, CXCR3 and STAT3 were measured by real-time PCR. (D-H) The protein levels of CXCL9, CXCR3, p-STAT3 and STAT3 were measured by western blot analysis. ${ }^{*} \mathrm{P}<0.05,{ }^{* *} \mathrm{P}<0.01$ and ${ }^{* * *} \mathrm{P}<0.001$ vs. control.

$43.8 \pm 0.25 \%$ and $54.6 \pm 0.41 \%$, respectively. These findings indicated that treatment with AGEs inhibited the proliferation of podocytes in a concentration- and time-dependent manner.

Effect of AGEs on the expression of CXCL9 and CXCR3, and STAT3 activation. To examine the effects of AGEs on the expression of CXCL9 and its receptor, and STAT3 activation in vitro, real-time PCR and western blot analysis were performed. The mRNA expression levels of CXCL9, CXCR3 and STAT3 were increased in the podocytes treated with AGEs (10, 50, 100 and $150 \mathrm{mg} / \mathrm{l})$ in a concentration-dependent manner compared with the controls (Fig. 3A-C). Similarly, the protein expression levels of CXCL9 and CXCR3 were increased, accompanied by the activation of STAT3; an increase in the levels of p-STAT3/STAT3 was observed in the AGE-treated podocytes in a concentration-dependent manner compared with the controls (Fig. 3D-H). Thus, AGEs at $150 \mathrm{mg} / \mathrm{l}$ were therefore used in the subsequent experiments. These data demonstrate that increased levels of CXCL9 and CXCR3, and
STAT3 activation may contribute to the AGE-induced inhibition of podocyte proliferation.

Knockdown of CXCL9 increases the proliferation and inhibits the apoptosis of podocytes. In order to examine the effects of CXCL9 on podocytes in vitro, CXCCL9 was knocked down by siRNA in podocytes. The results revealed that transfection with siNRA-CXCL9 significantly decreased the expression of CXCL9 in the AGE-treated podocytes at both the mRNA and protein level (Fig. 4A-C). In addition, the effects of CXCL9 on podocyte proliferation and apoptosis were also measured by CCK-8 assay and flow cytometry, respectively. Transfection with siNRA-CXCL9 significantly increased the proliferation and decreased the apoptosis of the AGE-treated podocytes compared with the cells treated with AGEs alone (Fig. 4D-F). However, the AGE-treated podocytes transfected with nonspecific siRNA (NC) exhibited no significant changes in CXCL9 expression, proliferation and apoptosis compared with the podocytes treated with AGEs alone. These results indicate 


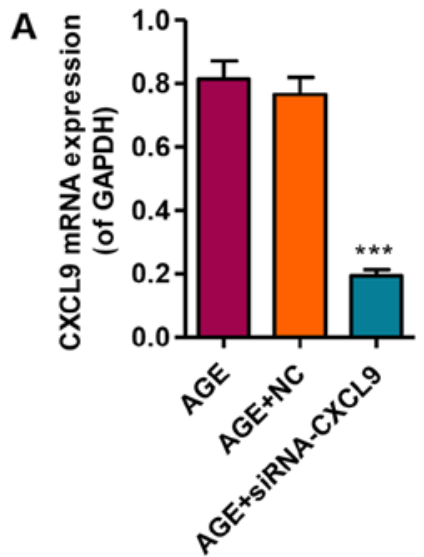

D

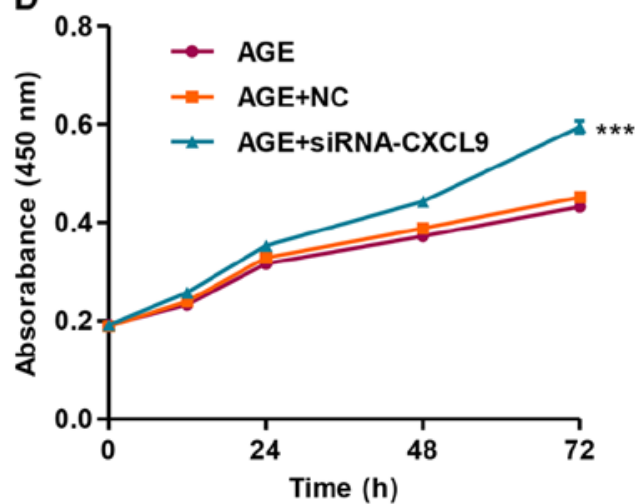

B

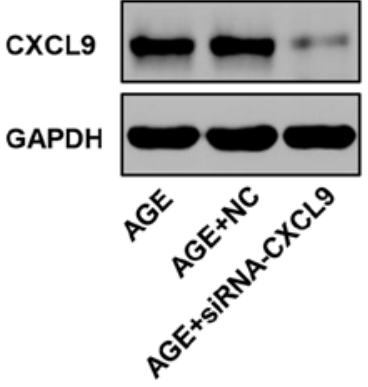

E
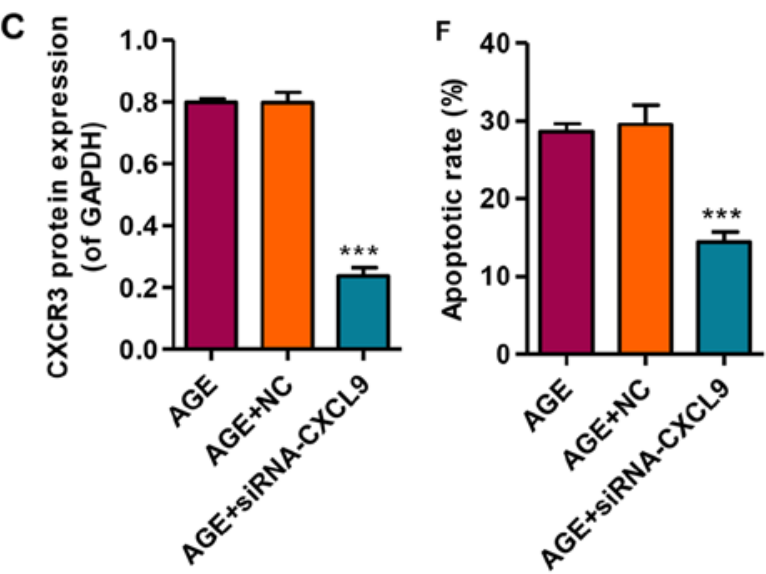
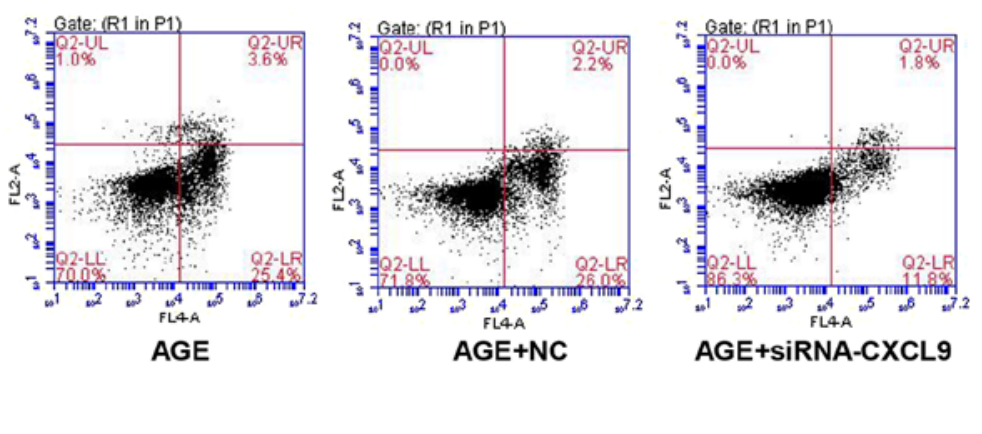

Figure 4. Effect of CXCL9 knockdown on the proliferation and apoptosis of podocytes. (A) The mRNA level of CXCL9 was measured by real-time PCR. (B and C) The protein level of CXCL9 was measured by western blot analysis. (D) The proliferation of podocytes was measured by cell counting kit-8 (CCK-8) assay at the indicated time points. ( $\mathrm{E}$ and $\mathrm{F}$ ) The apoptosis of podocytes was measured by flow cytometry. ${ }^{* * *} \mathrm{P}<0.001$ vs. AGE.
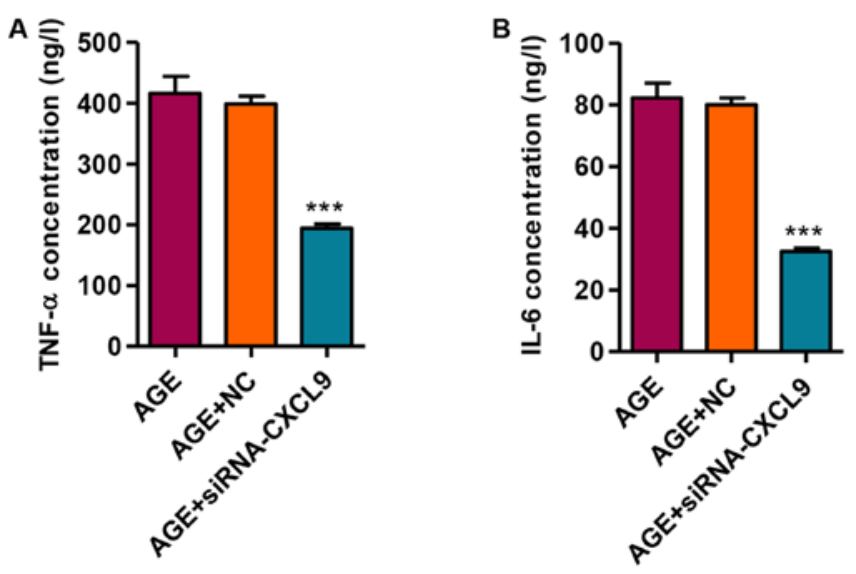

Figure 5. Effect of CXCL9 knockdown on the release of tumor necrosis factor- $\alpha$ (TNF- $\alpha$ ) and interleukin-6 (IL-6). The levels of (A) TNF- $\alpha$ and (B) IL-6 were measured by enzyme-linked immunosorbent assay (ELISA). **** $\mathrm{P}<0.001$ vs. AGE.

that CXCL9 is involved in the proliferation and apoptosis of AGE-treated mouse podocytes.

Knockdown of CXCL9 inhibits the release of the TNF- $\alpha$ and IL-6 inflammatory factors. We then measured the secretion levels of TNF- $\alpha$ and IL- 6 in response to transfection with siRNA-CXCL9 in the AGE-treated podocytes. Following the transfection of the AGE-treated podocytes with siRNA-CXCL9 for $48 \mathrm{~h}$, the secretion levels of TNF- $\alpha$ and IL-6 were significantly decreased (Fig. 5). These findings thus suggest that the downregulation of CXCL9 exerts an anti-inflammatory effect in AGE-damaged podocytes.

Effect of CXCL9 knockdown on protein expression and JAK2/ STAT3 activation. Following transfection of the podocytes with siRNA-CXCL9 for $48 \mathrm{~h}$, the expression levels of nephrin and podocin were significantly increased, while the Bax/Bcl-2 ratio and activated caspase-3 (act-caspase-3) were significantly suppressed (Fig. 6A and B). As we had found that STAT3 was activated in the AGE-treated podocytes, the levels of STAT3 and upstream JAK2 signaling were also measured by western blot analysis in the podocytes transfected wtih siRNA-CXCL9. Our results revealed that siRNA-CXCL9 significantly suppressed the activation of JAK2 and STAT3, as evidenced by decreased levels of p-JAK2/JAK2 and p-STAT3/ STAT3, in the AGE-treated podocytes transfected with siRNACXCL9 (Fig. 6C and D). These results thus suggest that the protective effects against AGE-induced damage to podocytes exerted by the downregulation CXCL9 are partially associated with the inhibition of JAK2/STAT3 activation in podocytes.

AG490 and valsartan attenuate the AGE-induced apoptosis of podocytes. Considering the role of JAK2/STAT3 signaling in AGE-induced damage to podocytes, the JAK2 inhibitor, AG490, was used. Valsartan, an angiotensin II receptor antagonist, has long been proven to exert an anti-proteinuria effect 
A

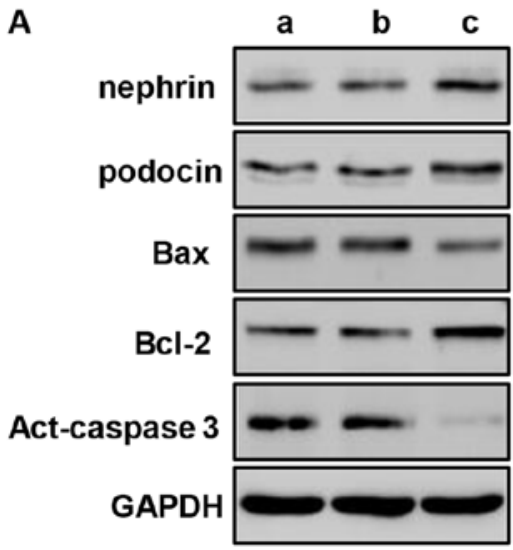

C

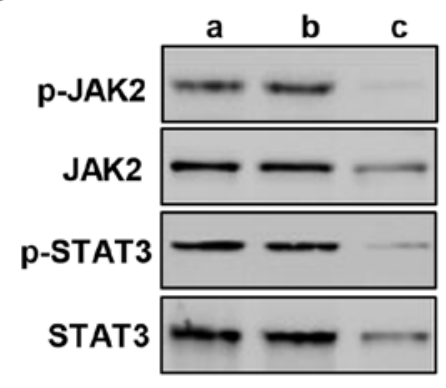

B
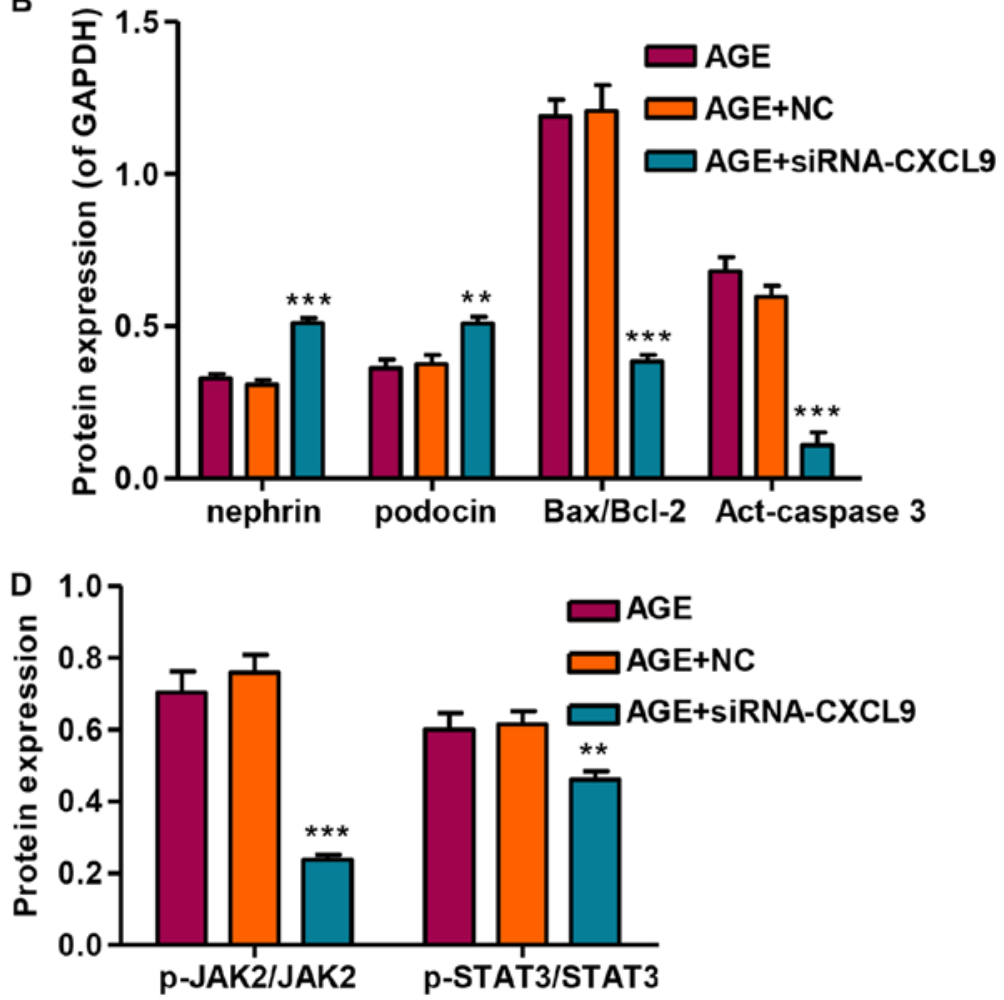

Figure 6. Effect of CXCL9 knockdown on protein expression and Janus kinase-2/signal transducer and activator of transcription 3 (JAK2/STAT3) activation. (A and B) The protein expression levels of podocyte markers and apoptosis-associated proteins were measured by western blot analysis. (C and D) The activation of JAK2 and STAT3 was measured by western blot analysis. lane a, AGE; lane b, AGE + NC; lane c, AGE + siRNA-CXCL9. ${ }^{* *} \mathrm{P}<0.01$ and ${ }^{* * *} \mathrm{P}<0.001$ vs. AGE.

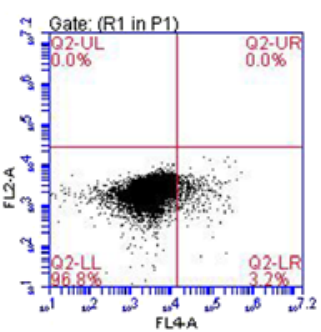

Control

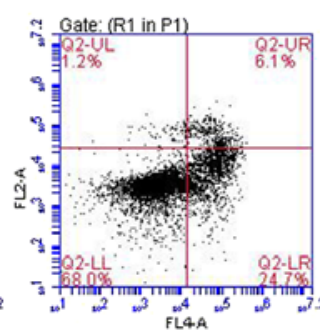

AGE

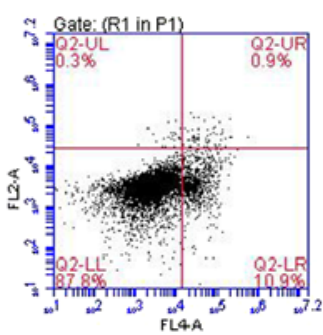

AGE+AG490

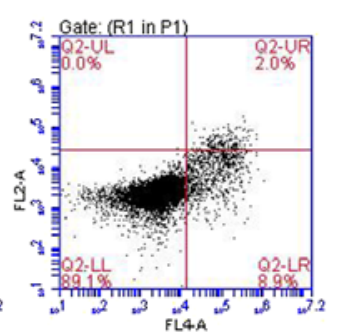

AGE+Valsartan

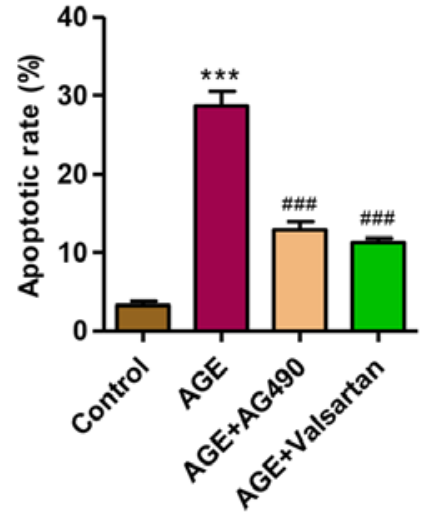

Figure 7. Effect of AG490 and valsartan on the apoptosis of podocytes. The apoptosis of podocytes was measured by flow cytometry. ${ }^{* * *}$ P $<0.001$ vs. control;

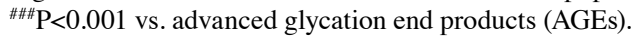

and to decrease podocytes damage in DN (37). Therefore, with valsartan as a control, we observed that following treatment of the podocytes with AGEs for $48 \mathrm{~h}$, the apoptotic rate was significantly increased. However, treatment with AG490 and valsartan markedly attenuated the apoptosis of the podocytes induced by treatment with AGEs (Fig. 7). These findings suggest that the activation of JAK2/STAT3 is implicated in AGE-induced podocyte apoptosis.

Effect of AG490 and valsartan on JAK2/STAT3 activation and protein expression. To investigate the role of AG490 in the apoptosis of podocytes induced by AGEs, the expression of CXCL9, Bax/Bcl-2 and activated caspase-3, as well as JAK2/STAT3 signaling was measured by western blot analysis. Following treatment of the podocytes with AGEs, the promoting effect of AGEs on JAK2 and STAT3 signaling in podocytes was markedly attenuated by treatment with AG490 and valsartan (Fig. 8A and B). The expression levels of CXCL9, Bax/Bcl-2 and activated caspase-3 were significantly increased following treatment with AGE; however, treatment with AG490 and valsartan markedly attenuated the overexpression of CXCL9, Bax/Bcl-2 and activated caspase-3 induced by AGE treatment (Fig. 8C and D). These results indicate that the AGE-induced apoptosis of podocytes is associated with the CXCL9-mediated activation of JAK2/ STAT3 signaling. 
A

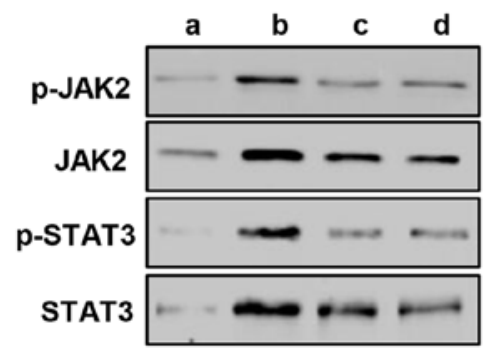

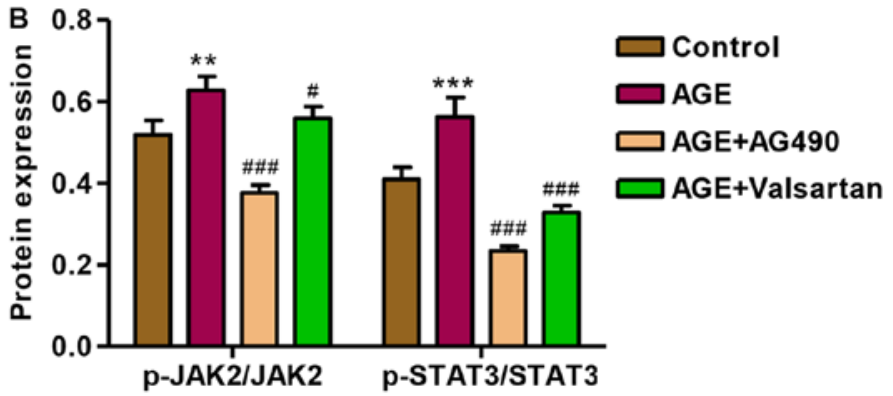

D

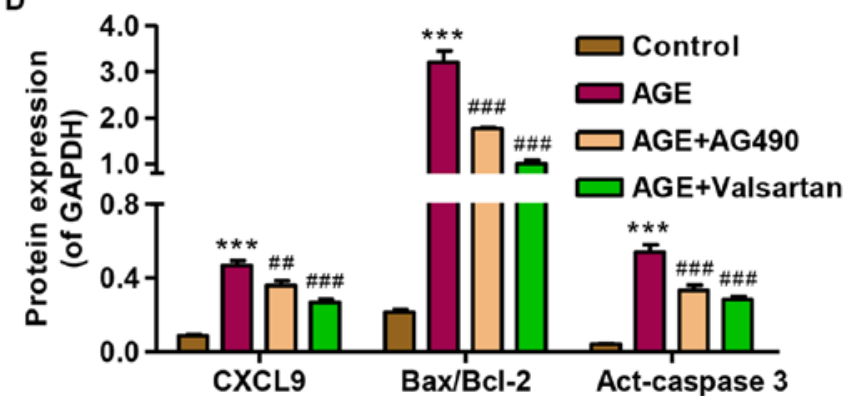

Figure 8. Effect of AG490 and valsartan on protein expression and Janus kinase-2/signal transducers and activator of transcription 3 (JAK2/STAT3) activation. (A and B) The activation of JAK2 and STAT3 was measured by western blot analysis. (C and D) The protein expression levels of CXCL9 and apoptosis-associated proteins were measured by western blot analysis. lane a, control; lane b, AGE; lane c, AGE + AG490; lane d, AGE + Valsartan. ${ }^{* * * *} \mathrm{P}<0.001 \mathrm{vs}$. control; ${ }^{*} \mathrm{P}<0.05$, ${ }^{\# \#} \mathrm{P}<0.01$ and ${ }^{\# \# \#} \mathrm{P}<0.001$ vs. advanced glycation end products (AGEs).

\section{Discussion}

CXC chemokines are particularly important for leukocyte infiltration in inflammatory diseases. It has been demonstrated that inflammation is one of the potential pathogenic mechanisms responsible for the development of DN (17). However, to date, there are limited data available on inflammation related to CXC chemokines in human DN. In this study, we measured the serum and urine levels of the CXC chemokine, CXCL9, in 45 patients wth $\mathrm{DN}$ and 45 healthy controls by ELISA. The serum and urine levels of CXCL9 in the patients with DN were significantly elevated compared with those in the controls. In agreement with our findings, a previous study found that the levels of 3 CXC chemokines, CXCL5, CXCL8 and CXCL9, were significantly increased in the urine and serum of patients with DN (18). These results suggest CXCL9 may be involved in the progression of DN.

In patients with type 1 and 2 diabetes, the serum and tissue levels of AGEs have been shown to be significantly increased compared with the healthy control subjects (19), and the tissue levels of AGEs in diabetic renal disease have been shown to be twice those of patients with only diabetes and no renal disease (20), suggesting that AGEs are responsible for the progression of DN. A reduction in podocyte density is an important determinant of progressive $\mathrm{DN}$ and precedes the development of renal dysfunction and albuminuria in diabetic patients and in animal models of diabetes $(21,22)$. Chen et al (23) reported that AGEs induced podocytes apoptosis in a dosedependent manner and Chuang et al (24) showed that AGEs activated FOXO4, leading to the apoptosis of podocytes, which was similar to our findings in that AGEs inhibited the proliferation of mouse podocytes in a dose- and time-dependent manner.
Accumulating evidence from animal models supports the notion that CXCL9 and its receptor, CXCR3, which is highly expressed in Th1 $\mathrm{CD}^{+}$cells, play a critical role in the recruitment of $\mathrm{T}$ cells, macrophages and dendritic cells during the development of chronic renal injury (25). An increase in CXCL9 protein levels was detected in streptozotocin-injected mice and showed its pronociceptive properties (26). Activated and resting CXCR3 macrophages express CXCR3 during kidney disease and are therefore central to inducing renal injury (12). In the present study, we found that CXCL9, as well as CXCR3, was significantly increased in response to AGEs in podocytes in a dose-dependent manner. Furthermore, STAT3 signaling was also activated by AGE treatment. After binding to their receptors, CXCL9 activates JAKs, which in turn leads to the tyrosine phosphorylation of STAT3 (27). Previous studies have reported that the increased expression of STAT3 reduces the IFN- $\alpha$ induction of CXCL9 mRNA in myeloid cells (28); in vitro, in human bronchial epithelial cells, IL-13 augmented IL-27-induced CXCL9 expression, which appeared to be due to augmented STAT1 activation and reduced STAT3 activation (29). The different stimulators, including AGEs, IL-27 and IFN- $\alpha$ may interpret the contradictory findings.

In this study, to further investigate the role of CXCL9 in AGE-induced podocyte damage, siRNA-CXCL9 was used to transfected into the podocytes. We found that CXCL9 downregulation significantly increased the proliferation and decreased the apoptosis of podocytes, and decreased the levels of the inflammatory factors, TNF- $\alpha$ and IL-6. In line with the experimental data, patients with type 2 diabetes have 3 -4-fold greater serum levels of TNF- $\alpha$ compared with nondiabetic patients, and that urinary TNF- $\alpha$ excretion correlates well with the clinical markers of $\mathrm{DN}$ and the progression 
of the disease (30). Experimental studies have indicated that IL- 6 overexpression in diabetic kidneys correlates with kidney hypertrophy and albumin excretion $(31,32)$. Nephrin and podocin are two recently discovered podocyte-specific proteins, pivotal in establishing podocyte slit membrane structure and in the maintenance of an intact filtration barrier (33). In the present study, nephrin and podocin expression levels were markedly increased following transfection of the AGEstreated podocytes with siRNA-CXCL9.x

Furthermore, the apoptosis-associated protein expression of Bax/Bcl-2 and activated caspase-3 was decreased, which is consistent with our apoptosis analysis measured by flow cytometry. In the present study, siRNA-CXCL9 triggered an effect opposite to that of the AGEs, whereby JAK2 and STAT3 activation was significantly suppressed in podocytes. Similar data by other investigators have indicated that increases in JAK2 and STAT3 gene expression occur in patients with DN compared with healthy controls (34). Lu et al (35) reported a mouse with reduced capacity of STAT3 activation showing less proteinuria, macrophage infiltration and inflammation at an early stage of DN. Total glucosides of paeony (TGP) significantly inhibited DN progression and these protective effects are associated with the ability of TGP to inhibit the JAK2/STAT3 pathway (13). AG490, a JAK2 specific inhibitor, was used in this study to determine the role of JAK2/STAT3 signaling in AGE-induced podocyte damage. Our results revealed that AG490 significantly inhibited JAK2 and STAT3 activation and the apoptosis of podocytes and the expression of CXCL9, Bax/Bcl-2, and activated caspase-3. Podocyte STAT3 activation can result in more severe nephropathy independent of upstream JAK signaling, or at least in changes in upstream JAK signaling. Thus, the activation of JAK2 and STAT3 in podocytes is important in the pathogenesis of DN (36). In addition, valsartan has been shown to exert protective effects against the progression of DN (37) and exerts a similar effect as AG490, suggesting that JAK2/STAT3 signaling is also implicated in the protective effects of valsartan against AGE-induced podocyte damage.

In conclusion, our study demonstrated that CXCL9 synthesis was upregulated in patients with DN and in AGE-treated mouse podocytes, and both CXCL9 downregulation and JAK2 inhibitor treatment significantly inhibited the decrease in proliferation and apoptosis induced by AGEs, as well as inflammatory factor secretion and JAK2/STAT3 activation. Our data may aid in the understanding of the multifactorial nature of DN, and suggest that CXCL9 may be a novel therapeutic target in the treatment of DN.

\section{References}

1. Remuzzi G, Schieppati A and Ruggenenti P: Clinical practice. Nephropathy in patients with type 2 diabetes. $N$ Engl J Med 346: 1145-1151,2002.

2. Yamagishi S and Matsui T: Advanced glycation end products, oxidative stress and diabetic nephropathy. Oxid Med Cell Longev 3: 101-108, 2010

3. Susztak K, Raff AC, Schiffer M and Böttinger EP: Glucoseinduced reactive oxygen species cause apoptosis of podocytes and podocyte depletion at the onset of diabetic nephropathy Diabetes 55: 225-233, 2006.

4. Navarro-González JF, Mora-Fernández C, Muros de Fuentes M and García-Pérez J: Inflammatory molecules and pathways in the pathogenesis of diabetic nephropathy. Nat Rev Nephrol 7: 327-340, 2011.
5. Koike N, Takamura $\mathrm{T}$ and Kaneko S: Induction of reactive oxygen species from isolated rat glomeruli by protein kinase $\mathrm{C}$ activation and TNF- $\alpha$ stimulation, and effects of a phosphodiesterase inhibitor. Life Sci 80: 1721-1728, 2007.

6. Dalla Vestra M, Mussap M, Gallina P, Bruseghin M,Cernigoi AM, Saller A, Plebani M and Fioretto P: Acute-phase markers of inflammation and glomerular structure in patients with type 2 diabetes. J Am Soc Nephrol 16 (Suppl 1): S78-S82, 2005.

7. Lim AK and Tesch GH: Inflammation in diabetic nephropathy. Mediators Inflamm 2012: 146154, 2012.

8. Yamagishi $\mathrm{S}$ and Imaizumi T: Diabetic vascular complications: Pathophysiology, biochemical basis and potential therapeutic strategy. Curr Pharm Des 11: 2279-2299, 2005.

9. Bohlender JM, Franke S, Stein G and Wolf G: Advanced glycation end products and the kidney. Am J Physiol Renal Physiol 289: F645-F659, 2005.

10. Busch M, Franke S, Rüster C and Wolf G: Advanced glycation end-products and the kidney. Eur J Clin Invest 40: 742-755, 2010.

11. Cheng C, Zheng Z, Shi C, Liu X, Ye Z and Lou T: Advanced glycation end-products reduce podocyte adhesion by activating the renin-angiotensin system and increasing integrin-linked kinase. Exp Ther Med 6: 1494-1498, 2013.

12. Menke J, Zeller GC, Kikawada E, Means TK, Huang XR, Lan HY, Lu B, Farber J, Luster AD and Kelley VR: CXCL9, but not CXCL10, promotes CXCR3-dependent immune-mediated kidney disease. J Am Soc Nephrol 19: 1177-1189, 2008.

13. Wang K, Wu YG, Su J, Zhang JJ, Zhang P and Qi XM: Total glucosides of paeony regulates JAK2/STAT3 activation and macrophage proliferation in diabetic rat kidneys. Am J Chin Med 40: 521-536, 2012.

14. Banes AK, Shaw S, Jenkins J, Redd H, Amiri F, Pollock DM and Marrero MB: Angiotensin II blockade prevents hyperglycemiainduced activation of JAK and STAT proteins in diabetic rat kidney glomeruli. Am J Physiol Renal Physiol 286: F653-F659, 2004.

15. Wang X, Shaw S, Amiri F, Eaton DC and Marrero MB: Inhibition of the Jak/STAT signaling pathway prevents the high glucose-induced increase in tgf-beta and fibronectin synthesis in mesangial cells. Diabetes 51: 3505-3509, 2002.

16. Jiao B, Wang YS, Cheng YN, Gao JJ and Zhang QZ: Valsartan attenuated oxidative stress, decreased MCP-1 and TGF- $\beta 1$ expression in glomerular mesangial and epithelial cells induced by high-glucose levels. Biosci Trends 5: 173-181, 2011.

17. Chung AC and Lan HY: Chemokines in renal injury. J Am Soc Nephrol 22: 802-809, 2011.

18. Higurashi M, Ohya Y, Joh K, Muraguchi M, Nishimura M, Terawaki H, Yagui K, Hashimoto N, Saito Y and Yamada K: Increased urinary levels of CXCL5, CXCL8 and CXCL9 in patients with type 2 diabetic nephropathy. J Diabetes Complications 23: 178-184, 2009.

19. Galler A, Müller G, Schinzel R, Kratzsch J, Kiess W and Münch G: Impact of metabolic control and serum lipids on the concentration of advanced glycation end products in the serum of children and adolescents with type 1 diabetes, as determined by fluorescence spectroscopy and nepsilon-(carboxymethyl)lysine ELISA. Diabetes Care 26: 2609-2615, 2003.

20. Genuth S, Sun W, Cleary P, Sell DR, Dahms W, Malone J, Sivitz W and Monnier VM; DCCT Skin Collagen Ancillary Study Group: Glycation and carboxymethyllysine levels in skin collagen predict the risk of future 10-year progression of diabetic retinopathy and nephropathy in the diabetes control and complications trial and epidemiology of diabetes interventions and complications participants with type 1 diabetes. Diabetes 54: 3103-3111, 2005.

21. Siu B, Saha J, Smoyer WE, Sullivan KA and Brosius FC III: Reduction in podocyte density as a pathologic feature in early diabetic nephropathy in rodents: Prevention by lipoic acid treatment. BMC Nephrol 7: 6, 2006.

22. Dai C, Stolz DB, Kiss LP, Monga SP, Holzman LB and Liu Y: Wnt/ $\beta$-catenin signaling promotes podocyte dysfunction and albuminuria. J Am Soc Nephrol 20: 1997-2008, 2009.

23. Chen Y, Liu CP, Xu KF, Mao XD, Lu YB, Fang L, Yang JW and Liu C: Effect of taurine-conjugated ursodeoxycholic acid on endoplasmic reticulum stress and apoptosis induced by advanced glycation end products in cultured mouse podocytes. Am J Nephrol 28: 1014-1022, 2008.

24. Chuang PY, Yu Q, Fang W, Uribarri J and He JC: Advanced glycation endproducts induce podocyte apoptosis by activation of the FOXO4 transcription factor. Kidney Int 72: 965-976, 2007.

25. Holdsworth SR and Tipping PG: Leukocytes in glomerular injury. Semin Immunopathol 29: 355-374, 2007. 
26. Zychowska M, Rojewska E, Pilat D and Mika J: The role of some chemokines from the CXC subfamily in a mouse model of diabetic neuropathy. J Diabetes Res 2015: 750182, 2015.

27. Huang JS, Lee YH, Chuang LY, Guh JY and Hwang JY: Cinnamaldehyde and nitric oxide attenuate advanced glycation end products - induced the JAK/STAT signaling in human renal tubular cells. J Cell Biochem 116: 1028-1038, 2015.

28. Ho HH and Ivashkiv LB: Role of STAT3 in type I interferon responses. Negative regulation of STAT1-dependent inflammatory gene activation. J Biol Chem 281: 14111-14118, 2006.

29. Xie M, Mustovich AT, Jiang Y, Trudeau JB, Ray A, Ray P, $\mathrm{Hu} \mathrm{H}$, Holguin F, Freeman B and Wenzel SE: IL-27 and type 2 immunity in asthmatic patients: Association with severity, CXCL9, and signal transducer and activator of transcription signaling. J Allergy Clin Immunol 135: 386-394, 2015.

30. Navarro JF, Mora C, Muros M and García J: Urinary tumour necrosis factor- $\alpha$ excretion independently correlates with clinical markers of glomerular and tubulointerstitial injury in type 2 diabetic patients. Nephrol Dial Transplant 21: 3428-3434, 2006.

31. Navarro JF, Milena FJ, Mora C, León C and García J: Renal proinflammatory cytokine gene expression in diabetic nephropathy: Effect of angiotensin-converting enzyme inhibition and pentoxifylline administration. Am J Nephrol 26: 562-570, 2006.
32. Thomson SC, Deng A, Bao D, Satriano J, Blantz RC and Vallon V: Ornithine decarboxylase, kidney size, and the tubular hypothesis of glomerular hyperfiltration in experimental diabetes. J Clin Invest 107: 217-224, 2001.

33. Saleem MA, Ni L, Witherden I, Tryggvason K, Ruotsalainen V, Mundel Pand Mathieson PW: Co-localization of nephrin, podocin, and the actin cytoskeleton: Evidence for a role in podocyte foot process formation. Am J Pathol 161: 1459-1466, 2002.

34. Brosius FC III and Alpers CE: New targets for treatment of diabetic nephropathy: What we have learned from animal models. Curr Opin Nephrol Hypertens 22: 17-25, 2013.

35. Lu TC, Wang ZH, Feng X, Chuang PY, Fang W, Shen Y, Levy DE, Xiong H, Chen N and He JC: Knockdown of Stat3 activity in vivo prevents diabetic glomerulopathy. Kidney Int 76: 63-71, 2009.

36. Brosius FC III and He JC: JAK inhibition and progressive kidney disease. Curr Opin Nephrol Hypertens 24: 88-95, 2015.

37. Zhang Y, Chen B, Hou XH, Guan GJ, Liu G, Liu HY and Li XG: Effects of mycophenolate mofetil, valsartan and their combined therapy on preventing podocyte loss in early stage of diabetic nephropathy in rats. Chin Med J (Engl) 120: 988-995, 2007. 